\title{
ON SECOND ZAGREB INDEX AND COINDEX OF SOME DERIVED GRAPHS
}

\author{
Bommanahal Basavanagoud ${ }^{1, *}$, Ivan Gutman ${ }^{2}$, \\ Chetana S. Gali ${ }^{1}$ \\ ${ }^{1}$ Department of Mathematics, Karnatak University, \\ Dharwad - 580 003, India \\ e-mail: b.basavanagoud@gmail.com, chetanagali19@gmail.com \\ ${ }^{2}$ Faculty of Science, University of Kragujevac, \\ P. O. Box 60, 34000 Kragujevac, Serbia, and \\ State University of Novi Pazar, Novi Pazar, Serbia \\ e-mail: gutman@kg.ac.rs
}

(Received March 30, 2015)

ABSTRACT. The second Zagreb index is defined as the sum of the products of the degrees of adjacent vertices. In this note, we examine the second Zagreb indices of some derived graphs and find expressions for these in terms of vertex degrees.

\section{Introduction}

In the previous paper [1] we have examined the Zagreb indices and coindices of several transformation graphs. There the relevance of such graphs for chemical applications was explained in due detail. In Ref. [1] also the basic facts on Zagreb indices and coindices were given, and appropriate literature sources for these topological indices quoted.

In this note we use the same notation and definitions as in $[1,6]$, and will therefore not repeat all their details. 
Thus, by $G$ is denoted a simple (molecular) graph with vertex set $V(G),|V(G)|=$ $n$ (= the order of $G$ ) and edge set $E(G),|E(G)|=m$ (= the size of $G$ ). If $u$ and $v$ are two adjacent vertices of $G$, then we write $u \sim v$; the edge connecting these vertices will be denoted by $u v$.

The degree of a vertex $v \in V(G)$ is the number of vertices adjacent to $v$ in $G$. It will be denoted by $d_{G}(v)$. The degree of an edge $e=u v$ in $G$, denoted by $d_{G}(e)$, is defined by $d_{G}(e)=d_{G}(u)+d_{G}(v)-2$.

The complement of the graph $G$, denoted by $\bar{G}$, is the graph with vertex $V(G)$, in which two vertices are adjacent if and only if they are not adjacent in $G$. Thus the size of $\bar{G}$ is $\left(\begin{array}{l}n \\ 2\end{array}\right)-m$ and $d_{\bar{G}}(v)=n-1-d_{G}(v)$ holds for all $v \in V(G)$.

The first and second Zagreb indices are defined as

$$
M_{1}(G)=\sum_{v \in V(G)} d_{G}(v)^{2} \quad \text { and } \quad M_{2}(G)=\sum_{u v \in E(G)} d_{G}(u) d_{G}(v)
$$

respectively. The first Zagreb index can also expressed as [3]

$$
M_{1}(G)=\sum_{u v \in E(G)}\left[d_{G}(u)+d_{G}(v)\right] .
$$

Došlić [2] has recently conceived the first and second Zagreb coindices as

$$
\overline{M_{1}}(G)=\sum_{u v \notin E(G)}\left[d_{G}(u)+d_{G}(v)\right] \quad \text { and } \quad \overline{M_{2}}(G)=\sum_{u v \notin E(G)} d_{G}(u) d_{G}(v)
$$

respectively.

The vertex-degree-based graph invariant

$$
F=F(G)=\sum_{w \in V(G)} d_{G}(w)^{3}
$$

was encountered in the paper [7], published in the 1970s, but was eventually completely ignored. Only quite recently there has been some interest to $F[5,6]$. For understandable reasons the name forgotten topological index was proposed for the $F$-index [5]. It is easy to show that $[4,5]$

$$
F(G)=\sum_{u v \in E(G)}\left[d_{G}(u)^{2}+d_{G}(v)^{2}\right]
$$

In the recent paper [6], several types of graph have been examined, which are derived from a parent graph $G$. These were the line graph $L=L(G)$, the subdivision 
graph $S=S(G)$, the edge-semitotal graph $T_{1}=T_{1}(G)$, the vertex-semitotal graph $T_{2}=T_{2}(G)$, the total graph $T=T(G)$ and the paraline graph $P L=P L(G)$. These derived graphs have been much studied in the earlier (both chemical and mathematical) literature, and their formal definitions are given in [6]. Here we provide just a self-explanatory illustration.

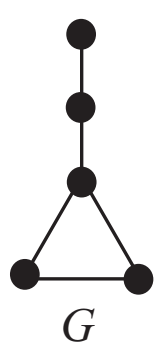

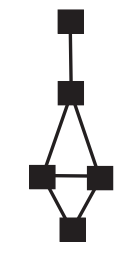

$L$

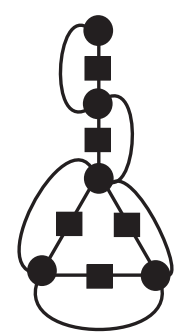

$T_{2}$

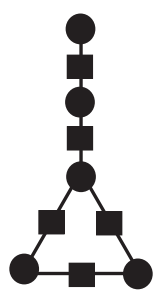

$S$

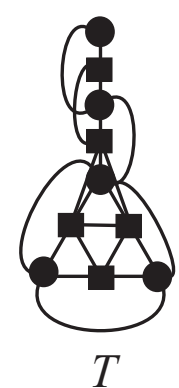

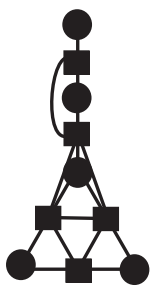

$T_{1}$

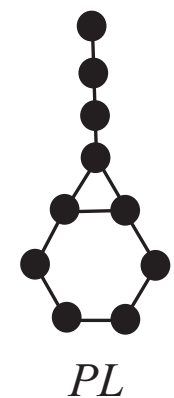

Fig. 1. Various graphs derived from the graph $G$. The vertices of these derived graphs (except the paraline graph $P L$ ), corresponding to the vertices of the parent graph $G$, are indicated by circles. The vertices of these graphs, corresponding to the edges of the parent graph $G$ are indicated by squares.

In what follows, in the considered derived graphs, the vertices of the derived graph corresponding to vertices of the parent graph (in Fig. 1 indicated by circles) will be referred to as $\gamma$-vertices. The vertices of the derived graph corresponding to edges of the parent graph (in Fig. 1 indicated by squares) will be referred to as $\lambda$-vertices [6]. 
In Ref. [6] expressions were derived for the first Zagreb indices of the above derived graphs:

Theorem 1.1. Let $G$ be a graph of order $n$ and size $m$. Then

$$
\begin{aligned}
M_{1}(L) & =F(G)-4 M_{1}(G)+2 M_{2}(G)+4 m \\
M_{1}(S) & =M_{1}(G)+4 m \\
M_{1}\left(T_{1}\right) & =F(G)+M_{1}(G)+2 M_{2}(G) \\
M_{1}\left(T_{2}\right) & =4 M_{1}(G)+4 m \\
M_{1}(T) & =F(G)+4 M_{1}(G)+2 M_{2}(G) \\
M_{1}(P L) & =F(G) .
\end{aligned}
$$

In Ref. [6], analogous expressions for the second Zagreb index have not been determined, and this remained a task for the future. We now fill this gap, and provide the missing expressions for the second Zagreb index.

The following relations are well known, see [6] and the references cited therein.

Theorem 1.2. Let $G$ be a graph of order $n$ and size $m$. Then

$$
\begin{aligned}
& M_{2}(\bar{G})=\frac{1}{2} n(n-1)^{3}-3 m(n-1)^{2}+2 m^{2}+\frac{2 n-3}{2} M_{1}(G)-M_{2}(G) \\
& \overline{M_{2}}(G)=2 m^{2}-\frac{1}{2} M_{1}(G)-M_{2}(G) \\
& \overline{M_{2}}(\bar{G})=m(n-1)^{2}-(n-1) M_{1}(G)+M_{2}(G) .
\end{aligned}
$$

From Theorem 1.2, it is clear that if $D \in\left\{L, S, T_{1}, T_{2}, T, P L\right\}$, and if $M_{1}(D)$ and $M_{2}(D)$ are known, then also $M_{2}(\bar{D}), \overline{M_{2}}(D)$, and $\overline{M_{2}}(\bar{D})$ are known. Therefore, since by Theorem 1.1, expressions for $M_{1}(D)$ are known, what really needs to be calculated are expression for $M_{2}(D)$. This we do in the subsequent section.

\section{Second Zagreb index of some derived graphs}

In order to determine the expression for the second Zagreb index of the line graph, we need to introduce a few auxiliary degree-based indices. 
Let $G$ be a (molecular) graph, and let $u, v$, and $w$ be its three vertices forming a path of length two. In other words, $u \sim v$ and $v \sim w$. Then we define three auxiliary Zagreb-type indices as follows:

$$
\begin{aligned}
M_{3}(G) & =\sum_{u v, v w \in E(G)} d_{G}(v)^{2} \\
M_{4}(G) & =\sum_{u v, v w \in E(G)}\left[d_{G}(u)+2 d_{G}(v)+d_{G}(w)\right] \\
M_{5}(G) & =\sum_{u v, v w \in E(G)}\left[d_{G}(u) d_{G}(v)+d_{G}(v) d_{G}(w)+d_{G}(w) d_{G}(u)\right]
\end{aligned}
$$

with summation going over all paths of length two, contained in the graph $G$.

Theorem 2.1. Let $G$ be a graph of order $n$ and size $m$. Then

$$
M_{2}(L)=2 M_{1}(G)+M_{3}(G)-2 M_{4}(G)+M_{5}(G)-4 m .
$$

Proof. Note that $L$ has $m$ vertices and $\frac{1}{2} M_{1}(G)-m$ edges.

The edge $e=u v$ of the graph $G$ is incident to $d_{G}(e)=d_{G}(u)+d_{G}(v)-2$ other edges of $G$. Therefore,

$$
\begin{aligned}
M_{2}(L) & =\sum_{x y \in E(L)} d_{L}(x) d_{L}(y)=\sum_{u v, v w \in E(G)}\left[d_{G}(u)+d_{G}(v)-2\right]\left[d_{G}(v)+d_{G}(w)-2\right] \\
& =\sum_{u v, v w \in E(G)}\left[4+d_{G}(u) d_{G}(v)+d_{G}(u) d_{G}(w)-2 d_{G}(u)+d_{G}(v)^{2}\right. \\
& \left.+d_{G}(v) d_{G}(w)-4 d_{G}(v)-2 d_{G}(w)\right]=4\left(\frac{1}{2} M_{1}(G)-m\right) \\
& +\sum_{u v, v w \in E(G)} d_{G}(v)^{2}-\sum_{u v, v w \in E(G)}\left[d_{G}(u)+2 d_{G}(v)+d_{G}(w)\right] \\
& +\sum_{u v, v w \in E(G)}\left[d_{G}(u) d_{G}(v)+d_{G}(v) d_{G}(w)+d_{G}(w) d_{G}(u)\right] .
\end{aligned}
$$

From Equations (1), (2) and (3), we get the expression (4) 
Theorem 2.2. Let $G$ be a graph of order $n$ and size $m$. Then $M_{2}(S)=2 M_{1}(G)$.

Proof. In the subdivision graph $S, d_{S}\left(v_{i}\right)=d_{G}\left(v_{i}\right), d_{S}\left(e_{i}\right)=2$ and $S$ has $n+m$ vertices and $2 m$ edges. Therefore,

$$
M_{2}(S)=\sum_{u v \in E(S)} d_{S}(u) d_{S}(v)
$$

where $u$ is a $\gamma$-vertex and $v$ is a $\lambda$-vertex. Further,

$$
M_{2}(S)=\sum_{u v \in E(S)} 2 d_{G}(u)=\sum_{u \in V(G)} d_{G}(u)\left[2 d_{G}(u)\right]=2 M_{1}(G)
$$

We now find expressions for the second Zagreb indices of $T_{1}$ and $T$ using reformulated Zagreb indices.

Milićević et al. [8] reformulated the Zagreb indices in terms of edge-degrees instead of vertex-degrees. The first and second reformulated Zagreb indices are defined respectively as

$$
E M_{1}(G)=\sum_{e \in E(G)} d_{G}(e)^{2} \quad \text { and } \quad E M_{2}(G)=\sum_{e \sim f} d_{G}(e) d_{G}(f)
$$

In the case of Zagreb indices, the transformation $G \rightarrow L(G)$ yields the reformulated Zagreb indices. Thus the original and the reformulated Zagreb indices are related as

$$
E M_{1}(G)=M_{1}(L(G)) \quad \text { and } \quad E M_{2}(G)=M_{2}(L(G))
$$

This is one way of expressing $M_{2}(L(G))$ in terms of edge-degrees.

Theorem 2.3. Let $G$ be a graph of order $n$ and size $m$. Then

$$
M_{2}\left(T_{1}\right)=2 E M_{1}(G)+E M_{2}(G)+2\left[M_{1}(G)+M_{2}(G)\right]+F(G)-4 m .
$$

Proof. In the edge-semitotal graph $T_{1}$, we have $d_{T_{1}}\left(v_{i}\right)=d_{G}\left(v_{i}\right), d_{T_{1}}\left(e_{i}\right)=d_{G}(u)+$ $d_{G}(v)$ and $T_{1}$ has $m+\frac{1}{2} M_{1}(G)$ edges. Therefore,

$$
M_{2}\left(T_{1}\right)=\sum_{u v \in E\left(T_{1}\right)} d_{T_{1}}(u) d_{T_{1}}(v)
$$




$$
\begin{aligned}
& =\sum_{u v \in E\left(T_{1}\right) \cap E(L)}\left[d_{T_{1}}(u) d_{T_{1}}(v)\right]+\sum_{u v \in E\left(T_{1}\right) \backslash E(L)}\left[d_{T_{1}}(u) d_{T_{1}}(v)\right] \\
& =\sum_{u=a b, v=b c \in E(G)}\left[d_{G}(a)+d_{G}(b)\right]\left[d_{G}(b)+d_{G}(c)\right] \\
& +\sum_{u w \in 2 E(G)} d_{G}(u)\left[d_{G}(u)+d_{G}(w)\right] \\
& =\sum_{e_{i} \sim e_{j} \in E(G)}\left[\left(d_{G}\left(e_{i}\right)+2\right)\left(d_{G}\left(e_{j}\right)+2\right)\right]+\sum_{u w \in E(G)}\left[d_{G}(u)+d_{G}(w)\right]^{2} \\
& =\sum_{e_{i} \sim e_{j}}\left\{d_{G}\left(e_{i}\right) d_{G}\left(e_{j}\right)+2\left[d_{G}\left(e_{i}\right)+d_{G}\left(e_{j}\right)\right]+4\right\}+F(G)+2 M_{2}(G) \\
& =2 E M_{1}(G)+E M_{2}(G)+4\left(\frac{1}{2} M_{1}(G)-m\right)+F(G)-4 m
\end{aligned}
$$

and Eq. (5) follows.

The expression for the second Zagreb index of the vertex-semitotal graph $T_{2}$ was obtained in [1]. We nevertheless state it for the sake of completeness:

Theorem 2.4. [1] Let $G$ be a graph of order $n$ and size $m$. Then

$$
M_{2}\left(T_{2}\right)=4\left[M_{1}(G)+M_{2}(G)\right] .
$$

Theorem 2.5. Let $G$ be a graph of order $n$ and size $m$. Then

$$
M_{2}(T)=2 M_{1}(G)+8 M_{2}(G)+2 E M_{1}(G)+E M_{2}(G)+2 F(G)-4 m .
$$

Proof. In the total graph $T$, we have $d_{T}\left(v_{i}\right)=2 d_{G}\left(v_{i}\right), d_{T}\left(e_{i}\right)=d_{G}(u)+d_{G}(v)$ and $T$ has $\frac{1}{2} M_{1}(G)+2 m$ edges. Therefore,

$$
\begin{aligned}
M_{2}(T) & =\sum_{u v \in E(T)} d_{T}(u) d_{T}(v)=\sum_{u v \in E(T) \cap E(G)} d_{T}(u) d_{T}(v) \\
& +\sum_{u v \in E(T) \cap E(L)} d_{T}(u) d_{T}(v)+\sum_{u v \in E(T) \backslash[E(G) \cup E(L)]} d_{T}(u) d_{T}(v) \\
& =\sum_{u v \in E(G)}\left[2 d_{G}(u)\right]\left[2 d_{G}(v)\right]+\sum_{e_{i} \sim e_{j} \in E(G)}\left[\left(d_{G}\left(e_{i}\right)+2\right)\left(d_{G}\left(e_{j}\right)+2\right)\right] \\
& +\sum_{u w \in 2 E(G)} d_{G}(u)\left[2 d_{G}(u)+2 d_{G}(w)\right]
\end{aligned}
$$




$$
\begin{aligned}
& =4 \sum_{u v \in E(G)} d_{G}(u) d_{G}(v)+\sum_{e_{i} \sim e_{j}}\left\{d_{G}\left(e_{i}\right) d_{G}\left(e_{j}\right)+2\left[d_{G}\left(e_{i}\right)+d_{G}\left(e_{j}\right)\right]+4\right\} \\
& +\sum_{u w \in E(G)}\left[2 d_{G}(u)+2 d_{G}(w)\right]\left[d_{G}(u)+d_{G}(w)\right] \\
& =4 M_{2}(G)+\left[2 E M_{1}(G)+E M_{2}(G)-4 m+2 M_{1}(G)\right]+2\left[F+2 M_{2}(G)\right]
\end{aligned}
$$

and Eq. (6) follows.

Theorem 2.6. Let $G$ be a graph of order $n$ and size $m$ with $p$ pendent vertices. Then

$$
M_{2}(P L)=M_{2}(G)-p+\sum_{u \in V(G)} d_{G}(u)^{d_{G}(u)}
$$

Proof. The paraline graph $P L$ of the graph $G$ has $2 m$ vertices, and the interesting property that $d_{G}(u)$ of its vertices have the same degree as the vertex $u$ of the parent graph $G$. Bearing this in mind, we have

$$
\begin{aligned}
M_{2}(P L) & =\sum_{u v \in E(P L)} d_{P L}(u) d_{P L}(v) \\
& =\sum_{u v \in E(P L) \cap E(G)} d_{P L}(u) d_{P L}(v)+\sum_{u v \in E(P L) \backslash E(G)} d_{P L}(u) d_{P L}(v) \\
& =\sum_{u v \in E(G)} d_{G}(u) d_{G}(v)+\sum_{u \in V(G)} d_{G}(u)^{d_{G}(u)}-p
\end{aligned}
$$

and Eq. (7) follows.

Acknowledgement. ${ }^{*}$ This research is supported by UGC-SAP DRS-II New Delhi, India: for 2010-2015. ${ }^{1}$ This research is supported by UGC-UPE (Non-NET)-Fellowship, K. U. Dharwad, No. KU/Sch/UGC-UPE/2014-15/895, dated: 24 Nov 2014.

\section{REFERENCES}

[1] B. Basavanagoud, I. Gutman, V. R. Desai, Zagreb indices of generalized transformation graphs and their complements, Kragujevac J. Sci. 37 (2015) 99-112.

[2] T. Došlić, Vertex-weighted Wiener polynomials for composite graphs, Ars Math. Contemp. 1 (2008) 66-80. 
[3] T. Došlić, B. Furtula, A. Graovac, I. Gutman, S. Moradi, Z. Yarahmadi, On vertex-degree-based molecular structure descriptors, MATCH Commun. Math. Comput. Chem. 66 (2011) 613-626.

[4] T. Došlić, T. Réti, D. Vukičević, On the vertex degree indices of connected graphs, Chem. Phys. Lett. 512 (2011) 283-286.

[5] B. Furtula, I. Gutman, A forgotten topological index, J. Math. Chem. 53 (2015) 1184-1190.

[6] I. Gutman, B. Furtula, Z. Kovijanić Vukićević, G. Popivoda, Zagreb indices and coindices, MATCH Commun. Math. Comput. Chem. 74 (2015) 5-16.

[7] I. Gutman, N. Trinajstić, Graph theory and molecular orbitals, Total $\pi$-electron energy of alternant hydrocarbons, Chem. Phys. Lett. 17 (1972) 535-538.

[8] A. Miličević, S. Nikolić, N. Trinajstić, On reformulated Zagreb indices, Mol. Divers. 8 (2004) 393-399. 